the surrounding boulder clay has a normal low percentage; there are great differences in the percentages of lime and soluble iron, and also differences in the sedimentary petrology, in particular the percentage of dark minerals and the higher percentage of amphiboles, and the differences in the grain-size.

These differences indicate that these blocks of boulder clay have no connexion with the normal surrounding boulder clay. They must therefore have another origin. In view of the shape and the position in the normal boulder clay, it is clear that these are lenses or floes, composed of a different sort of boulder clay from the normal. It is probable that these floes are parts of another glacial deposit torn loose, embedded in the ground moraine and transported by the ice-cover during the Riss glaciation.

I have tried to find out from which glaciation these floes may have been formed. Indications of their origin may be the content of erratics. Both the east Baltic sedimentary rocks and the high-percentage Rapakivi rocks indicate a Mindel age. They resemble the autochthonic glacial deposits of the Mindel glaciation in the north of Germany.

In addition to the glacial areas on the bottom of the north-east of the former Zuyder Zee, I found these boulder clay floes in several different parts, about eighty altogether, of the north and east Netherlands. I should expect to find these floes in the adjoining Riss deposits in the north-east of Germany too. Their presence in the normal Riss deposits finally explains the abnormal east Baltic associations of erratics and the unusual colour differences of the boulder clay.

A detailed report on geological aspects of the existence, contents and spread of these boulder clay floes in the Netherlands will be published shortly.

\section{BOWLING-GREENS AND BOWLS}

\section{By J. H. COLE and DR. W. LAWRENCE BALLS, F.R.S.}

$I^{\mathbb{N}}$ Egypt, South Africa, and part of the United States, the bowling-greens have to be made with species of Cynodon (crested dogs-tail) grass, in order that they may stand up to summer heat. This grass, known to us in two or three species and many varieties, has a habit of one-sided growth, which imparts a one-way 'nap' to the playing surface, not only on bowling-greens but on putting-greens also. Both bowlers and golfers in these countries are used to seeing the ball run as if the whole surface of the green were sloping, though it may be dead level. The intensity of the effect varies from one strain to another: the Indian 'dhob' is the worst we have met; the less coarse Egyptian 'negeel' and the quarterscale $C$. transvaalensis (called 'Uganda' in Egypt) both behave alike, though the latter is so much finer in texture as a lawn. On the other hand, a strain which is nap-free, known as 'Royal Cape', was isolated by Dr. Murray near Cape Town. We have checked this (with Mr. R. P. Black) in propagation plots at the Gezira Sporting Club in Cairo; it is, however, quite exceptional, and not easy to establish in Egypt.

The direction in which the nap points, and its intensity, can be measured in several ways. The earliest we used, and slowest but most direct, is to roll a ball of any kind down a chute towards all points of the compass in succession, and plot a polar diagram of the distances reached in each direction. The quickest, and most useful practically, since it will deal with a single square foot in each reading, is to oscillate a smooth six-inch disk of wood on the grass surface, noting the direction in which it moves, and how quickly; this can be done easily and accurately by using a light tripod about four feet high with an operating handle at the top, and a light duraluminium tube with universal joints connecting the disk to the handle at the top, without introducing any lateral displacement couple. Even now we are in doubt as to the components of the nap; it is not entirely superficial, since a green may show more nap after mowing than it had when uncut; we have even found traces of nap in a different direction under the predominant one. It would seem that the root-stoeks of the grass build up a 'geological' structure recording the history of the nap.

Our earliest observations at the Gezira Sporting Club showed that the nap in 1930, under the regime of grass-control then employed, had a general trend to the north-west. Under our present regime of heavy manuring (sulphate of ammonia) and hard cutting, it points mostly west of south. The startingpoint, with zero nap, is a lawn which has been 'skinned' of its grass-cover, and allowed to regenerate from the root-stocks. Six months later we have seen a slight westward nap in the late winter, a south. ward nap by the late summer, and finally a settling. down to south-west. But the settling-down is incomplete, and lawns which have not been skinned for years will still show changes over a swing of some seventy degrees, under the same regime, as the seasons change. Obviously the cause of nap is complex, but it would seem that its direction is determined primarily by water-strain, the precise development of the reaction thereto being determined by the balanee of soil-moisture, manuring, and weather conditions. It need scarcely be added that the system of mowing is so arranged as to eliminate that possible cause completely.

Very strange bowl-tracks can be obtained when playing on such nappy lawns with the wrong kind of bowl, or when using the testing-chute. An English 'standard bowl' may take two feet of bias against the nap, but six feet with the nap, if the chute is set at right angles to the nap-direction. If set to bowl up and down the nap-direction, it may give even bias in both hands, but run several yards farther with the nap than against it. The effect on the muscular effort of the bowler is the same as if the green-surface were on the slope.

Bowls of modified shape give still stranger results. A flat track only half an inch in width, cut on the tread of a normal bowl, will make it entirely independent of the gravitational bias given it by its maker. Like a skater rocking from inside to outside edge, and back again, it will take a sigmoid course as the nap along its track changes direction locally. On a green with uniform and strong nap it is almost impossible to guess from its course which bias had been given; the nap takes complete control. As a limit case of this, instead of the cylindrical track, we can make a frustum of a cone, wider than it is high, when lying on its side; the path of this at low speed, or on a napless green, must be an arc of a circle, there being no gravitational bias; but if bowled fast on a nappy green against the nap it will turn a somersault.

It was noticed that bowls constructed by different makers were affected in varying degree. One Aus. 
tralian make in particular was almost completely insensitive to cross-nap, while another make, an English one, had a tendency to reverse the napeffect, taking more bias against the nap and less with it. Measurements on these and other bowls with a dial micrometer showed that the less nap-sensitive was the bowl, the more closely it approximated to a spherical form along its actual running-contact tread.

Conversely, the bowls which were most affected, sometimes fantastically, were found to have a radius of curvature across the tread which was much greater than the radius along the tread; for example, a 5 in. bowl, $2 \frac{1}{2}$ in. along the tread, might be $2 \frac{5}{8}$ in. across the tread. The running tread is only about an inch wide, so that such a bowl could be made spherical-treaded by removing only about 0.005 in. at the most. Steps were taken to correct the treads on a set of bowls which were badly affected, but since the re-shaping depended on cutting to limits well inside 0.001 in., it looked like a tool-maker's job to centre each bowl correctly in a lathe. This was practically impossible, when some fifty sets, and more, of club bowls were concerned. It was found, however, that a simple cradle arrangement over a mandrel, with a ball-bearing back-stop and lateral shims between bowl and cradle, gave sufficient drive to allow the use of a scraper. Several sets of bowls were thus converted, taking about half an hour per bowl; they were picked out of the cradle at intervals and tested across the track to a gauge of the same curvature as that which would fit along the track. The lathe was replaced by a sewing-machine stand, and the demand for re-shaping in the Gezira Sporting Club exceeds the supply of willing skilled operatives.

The cause of the reaction of the bowl to the nap is at present uncertain, just as is the causation of the nap itself. But observations show that bowls running across the nap show a marked tilt of their axis of rotation, which may be deflected from the horizontal by as much as $20^{\circ}$. The axis is tilted up in the direction of the nap, just as if the pile of the grass were acting as a banking, and the bowl were running on the banking. We suspect that the effect on the bias is due to this tilting of the axis of rotation (see accompanying diagram).
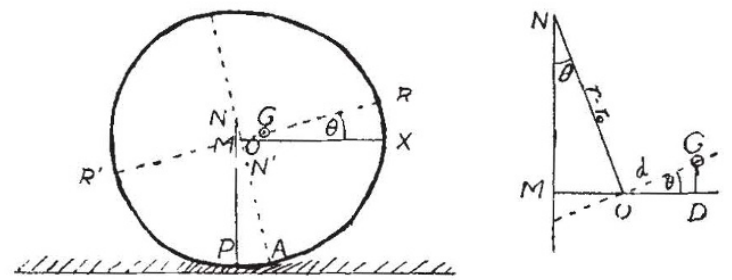

SECTION ACROSS TRACK, BIAS BEING SET WITH THE NAP.

In the above figure, $P$ is the centre of pressure on the grass, but owing to nap the point of maximum grip on the grass is at $A$, hence the tilt of the axis of rotation.

$O$, centre of bowl ; $G$, centre of gravity ; $N$, centre of curvature of section at $P-A ; P N$, perpendicular to the ground at $P ; O M$, perpendicular from $O$ on $P N ; \theta$, angle of slope $=\angle M N O=\angle R O X ; R O R^{\prime}$, axis of rotation; $O A=r_{0} ; N P=r=N A ; \therefore$ $O N=r-r_{0} ; O G=d$.

$\therefore M O D=M O+O D=\left(r-r_{0}\right) \sin \theta+d \cos \theta$.

$\therefore$ increase of bias distance $=M D-O G$, which is equal to $\left(r-r_{0}\right) \sin \theta-d(1-\cos \theta)$.

For $\theta=20^{\circ}, \sin \theta=0.342$, and $1-\cos \theta=0.060$.
Since $d$ is of the order $0 \cdot 1$ in., then $d(1-\cos \theta)$ is of the order $0.006 \mathrm{in.}$, and so may be neglected in comparison with $\left(r-r_{0}\right) \sin \theta$, unless $r=r_{0}$.

The inclination of the spherical bowl is such as to set its median plane at right angles to the slope of the grass blades, in both directions and on both 'hands'. We omit any consideration of gyroscopic effects, which must exist at the beginning of the run of a bowl. Apart from this, the causes of bias are three-fold: first, the gravitational cause due to lateral asymmetry of the bowl; secondly, the shape cause, as exaggerated in the case of a conical 'bowl' ; thirdly, the asymmetry of the lawn structure, as described above. English bowling-greens seem to be free from the latter complication; casual examination seems to indicate that similar one-sided growth happens in our English grasses and climate, but that it is random and localized to an inch or so, the end result being practical symmetry of grass-surface.

We hope that this communication may direct the attention of plant physiologists to an interesting problem, both in the comparison of Cynodon with other grasses, and in the comparison of varied ecological conditions on various grasses. We also hope that it may save some 'greens stewards' being blamed for levelling errors which have no existence.

\section{ACCELERATION OF CHARGED PARTICLES}

1 'HE two most successful methods for accelerating charged particles to very high energies are those employed in the cyclotron and betatron. In the eyclotron, if a very large number of individual accelerations is required, there may be difficulty in keeping the particles in step with the applied oscillating electric field, especially when relativistic mass change causes an appreciable variation in the angular velocity of the particles.

E. M. McMillan (Phys. Rev., 68, 143; 1945) has proposed a device, which because of the similarity in its behaviour to that of a synchronous motor, he has called a 'synchroton'. It is shown that a particle, the energy of which (called its 'equilibrium energy') is such that its angular velocity matches the frequency of the electric field, moves in a stable stationary orbit, and any displaced orbit, due to displacement in phase or energy of the particle, will tend to correct itself. In order to accelerate the particles, the value of the equilibrium energy must be changed by varying either the magnetic field or the frequency. The practical application of the method depends on the type of particles to be accelerated. In the case of electrons, the equilibrium value of the energy varies during the acceleration by a large factor and it is more practical to vary the magnetic field; for heavy-particle acceleration, the frequency may be varied. A possible design for such a $300-\mathrm{MeV}$. accelerator is outlined, and the construction at the Radiation Laboratory of the University of California at Berkeley is stated to have been planned.

D. W. Kerst (Phys. Rev., 68, 233 ; 1945) has also outlined a method for increasing the energies of particles obtained by the betatron. The betatrons used to date have all followed the design of the original 1941 Kerst model (see Nature, January 26, p. 90), in which the flux density at the orbit of the electron is limited to a value less than half the flux density which can be used at the centre of the orbit 